\title{
Experimental Test and Analytical Modeling of Mechanical Properties of Graphene-Oxide Cement Composites
}

\section{Zhongcheng Duan ${ }^{1,2}$, Li Zhang ${ }^{3}$, Zhiyuan Lin $^{2}$, Ding Fan ${ }^{2}$, Mohamed Saafi ${ }^{4}$, João Castro Gomes ${ }^{5}$ and Shangtong Yang ${ }^{2}$ *}

${ }^{1}$ School of Architecture and Design, China University of Mining and Technology, Xuzhou, 221116, China

${ }^{2}$ Department of Civil and Environmental Engineering, University of Strathclyde, Glasgow, G1 1XJ, United Kingdom

${ }^{3}$ Department of Architecture and Building Environment, the University of Nottingham, Nottingham, NG7 2RD, Unite Kingdom

${ }^{4}$ Department of Engineering, Lancaster University, Lancaster, LA1 4YW, United Kingdom

${ }^{5}$ Department of Civil Engineering and Architecture, University of Beira Interior, Covilha, 6201-001, Portugal

\section{ABSTRACT}

Graphene oxide (GO) has recently been considered as an ideal candidate for enhancing the mechanical properties of the cement due to its good dispersion property and high surface area. Much of work has been done on experimentally investigating the mechanical properties of GO-cementitious composites; but there are currently no models for accurate estimation of their mechanical properties, making proper analysis and design of GO-cement based materials a major challenge. This paper attempts to develop a novel multi-scale analytical model for predicting the elastic modulus of GO-cement taking into account the GO/cement ratio, porosity and mechanical properties of different phases. This model employs Eshelby tensor and Mori-Tanaka solution in the process of upscaling the elastic properties of GO-cement through different length scales. In-situ micro bending tests were conducted to elucidate the behavior of the GO-cement composites and verify the proposed model. The obtained results showed that the addition of GO can change the morphology and enhance the mechanical properties of the cement. The developed model can be used as a tool to determine the elastic properties of GO-cement through different length scales.

KEYWORDS: Multi-scale modelling; Graphene-Oxide; Elastic properties; Cementitious materials; Upscaling; In-situ SEM test.

* Corresponding authors: shangtong.yang@ @strath.ac.uk (s.yang). 


\section{INTRODUCTION}

Nanomaterials have recently attracted considerable attention for their application to cementitious composites, for improving the properties of cement/concrete. A wide range of nanomaterials such as metal oxide or silica ${ }^{1}$, nanofibers ${ }^{2}$, nanotubes ${ }^{3}$ and graphene ${ }^{4}$ have been added into cementitious materials to improve their compressive and flexural strength, Young's modulus and other microstructure properties. Graphene is a single atom thick sheet of hexagonally arranged carbon atoms with a carbon-carbon distance of $0.142 \mathrm{~nm}^{5}$. It is about more than 100 times stronger than steel by weight, conducting heat and electricity efficiently and nearly transparent ${ }^{5}$. However, there is an issue of dispersion when mixing with cementitious materials. Graphene oxide (GO), a product of chemical exfoliation of graphene, can be an excellent nano reinforcement for cementitious nanocomposites, due to its good dispensability in water, high aspect geometric ratio and excellent mechanical properties.

Previous studies have revealed that GO shows better adhesion bonding properties to the matrix than graphene, as a result of its oxygen functional groups provided ${ }^{6,7}$. Moreover, GO is hydrophilic due to the carbonyl, epoxide, carboxyl and hydroxyl groups on its surface thus making it highly dispersible in water as a result ${ }^{8}$. Because of these functional groups, GO can react with cement and provide strong stress transfer capacity which enables higher stress transfer between the matrix and the GO, thereby increasing the stiffness of the plain matrix ${ }^{9}$. Literature review suggests that GO can increase the 28-day compressive and flexural strength of cement by as much as $72.7 \%$ and $67.1 \%$, respectively at a GO concentration of $0.06 \mathrm{wt} \%$ and $0.04 \mathrm{wt} \%$, respectively ${ }^{9}$. Saafi et al. ${ }^{4}$ investigated the material and mechanical properties of grapheme/fly ash geopolymeric composite cement and experimentally quantified the enhancement in these properties.

GO can influence the hydration process of cement and alter the microstructure of cement paste. The 
hydration rate of cement has been found to increase as the GO content increases, due to nucleation effect ${ }^{10}$. However, GO agglomeration can affect the increase of hydration rate of cement ${ }^{11}$. GO can also densify cement paste, leading to less porosity and more hydration products; in particular, GO can promote the production of rod-like and needle-like hydration products and their further assembly into regular flower-like or polyhedron-like products, resulting in final formation of a denser microstructure ${ }^{9}$. Moreover, the workability of GO-cement can be improved by $21 \%$ with the addition of $0.03 \mathrm{wt} \% \mathrm{GO}^{11}$. Further, the electrical resistivity of GO-cement can be affected by GO but it varies for different hydration stages and GO dosages ${ }^{10}$.

Research activities on modeling of GO/cement composites are scarce and to date, most of the models were developed for GO/polymer and carbon nanotube/polymer (CNTRP) composites. A multi-scale modeling approach to extract mechanical properties of CNTRP was developed. ${ }^{12,13}$ The model considered effective parameters associated with meso- and micro-scale, non-uniform dispersion of CNTs and non-perfect bounding provided by van der Waals interaction. Both agglomerated and fully dispersed CNTs were formulated in the model. Moreover, the influences of carbon nanotube waviness on the stiffness reduction of CNTRP composites were investigated ${ }^{14}$. The structural and mechanical properties of the graphene/GO based polymer composites were investigated through molecular dynamics simulations ${ }^{15}$. It was found that the interlayer spacing and the layer-matrix interactions control the large scale properties of these composites, thereby affecting the elastic modulus of the composites. Moreover, the chemical composition of individual GO sheets also affects the mechanical properties and the elastic moduli of individual GO sheets decrease with a higher density of oxygen-containing groups. To the authors' knowledge, there is no model for the prediction of the mechanical properties of GO-cement. As a result, proper analysis and design of GO-cement based materials remain a major challenge and hinders the application of GO cementitious composites in civil engineering. 
This paper attempts to experimentally investigate the properties of GO-cement and develop a multi-scale analytical model for the elastic modulus of the GO reinforced cement, which is a key material parameter representing the elasticity of material. Volume fractions and mechanical properties of different phases at various length scales are considered in the formulation of the macroscale Young's modulus. Voids have also been considered in the formulation by assuming zero stiffness of them. In-situ SEM three-point bending tests were carried out to understand the fracture mechanisms of GO-cement composites and verify the derived model by comparing the Young's modulus obtained from both modeling and experimental tests. Mercury Intrusion Porosimetry (MIP) tests are also carried out to find out the effects of adding GO on the porosity of cement. Some key parameters such as the bulk and shear moduli of GO and Calcium Silicate Hydrates $(\mathrm{C}-\mathrm{S}-\mathrm{H})$ were chosen and their effects on the macroscale Young's modulus have been investigated. The developed model can be used as a useful tool for predicting the Young's modulus of GO-cement with a variety of composition and fractions. Accurate prediction of the mechanical properties of GO-cement will help accelerate the application of GO into civil engineering industries.

\section{MODEL FORMULATION}

In this paper, GO-cement composites are studied at 3 different length scales, as shown in Figure 1. At microscale, there are mainly GO and C-S-H while at mesoscale, calcium cydroxide $(\mathrm{CH})$, voids and some unhydrated cement ${ }^{16,17}$. To determine the scales in multi-scale modelling, the structural dimensions need to be significantly larger than the dimensions of the material in homogeneities. Accordingly, Representative Volume Element (RVE) is introduced to represent the basic unit of homogeneity. Eshelby tensor and Mori-Tanaka solution are employed in the process of upscaling the elastic properties of GO-cement through different length scales. There is an argument that the GO is a disk-like material while Mori-Tanaka may not be directly applicable. However, in practice, 

a few layers of GO always stack together or fold, forming a roughly spherical shape, as shown in Figure 2. It is almost impossible to have a single layer of disk-like GO sheet in cement and the application of Mori-Tanaka scheme can therefore be justified. This justification is evident in some previous work, e.g., Alkhateb et al. ${ }^{18}$ and Noh et al. ${ }^{19}$. RVE is utilized which is essential to find the homogenized constitutive response at each smaller scales ${ }^{20,21}$. In this paper, an RVE with a size of $50 \times 50 \mu \mathrm{m}$ is chosen for the two-phase matrix-inclusion geometries at the microscale level. At this level, the RVE is displayed in Figure 3. The average stain of the RVE is equal to the homogenized strain.

At microscale, C-S-H is considered as the matrix and GO as the inclusion. As shown in Figure 3. The matrix phase has a volume $V_{m}$ and a volume fraction $f_{m}$. The volume for the inclusion phase is $V_{i}$ and the volume fraction is $f_{i}$. The relationships between these parameters can be expressed as follows,

$$
\begin{gathered}
f_{m}=\frac{V_{m}}{V_{p}} \\
f_{i}=\frac{V_{i}}{V_{p}} \\
f_{m}+f_{i}=1
\end{gathered}
$$

In order to work out the stress and strain fields in the RVE, the mechanical response of each phase needs be determined by assuming each constituent phase follows its own constitutive relations. The continuum mechanism can then be applied to describe the relation between the average stress and strain in the sub-domains of the RVE, presented as follows,

$$
\bar{\sigma}=C_{p} \bar{\varepsilon}
$$

where $\boldsymbol{C}_{\boldsymbol{p}}$ is the Elasticity tensor and determines the stress-strain relationship for each phase 
individually.

127

128

129

130

131

132

133

134

135

136

To relate the stresses/strains across phases, concentration tensors are introduced. In linear continuum micromechanics, the homogenized strain $\bar{\varepsilon}$ can be linked to the individual strain $\varepsilon_{r}$ at different phase by means of a linear strain localization condition which can be shown as follows:

$$
\varepsilon_{r}=\boldsymbol{A}_{r}: \bar{\varepsilon}
$$

where $\boldsymbol{A}_{\boldsymbol{r}}$ is the fourth-order concentration (localization) tensor.

By introducing the fourth-order unit tensor $I=\frac{\partial \varepsilon_{i j}}{\partial \varepsilon}, A_{r}$ can be expressed as follows:

$$
<\boldsymbol{A}_{r}>_{V_{p}}=\boldsymbol{I} \quad \text { or } \quad f_{m} \boldsymbol{A}_{\boldsymbol{m}}+f_{i} \boldsymbol{A}_{\boldsymbol{i}}=\boldsymbol{I}
$$

where $A_{m}$ and $A_{i}$ are the $4^{\text {th }}$ order localization tensor for matrix and inclusion respectively. Therefore, $\boldsymbol{A}_{\boldsymbol{m}}$ can be expressed as follows.

$$
A_{m}=\frac{\left[I-\mathrm{f}_{\mathrm{i}} A_{i}\right]}{\mathrm{f}_{\mathrm{m}}}
$$

By introducing the linear elastic constitutive relation for different phases contained in $V_{p}$, the linear homogenization formula for the macroscopic elasticity tensor can be determined as follows:

$$
\boldsymbol{C}_{\boldsymbol{h}}=\left\langle\boldsymbol{C}: \boldsymbol{A}>=\sum_{r} f_{r} \boldsymbol{C}_{\boldsymbol{r}}: \boldsymbol{A}_{\boldsymbol{r}}=f_{m} \boldsymbol{C}_{\boldsymbol{m}}: \boldsymbol{A}_{\boldsymbol{m}}+f_{i} \boldsymbol{C}_{\boldsymbol{i}}: \boldsymbol{A}_{\boldsymbol{i}}\right.
$$

According to Mori-Tanaka theory ${ }^{21}$ the localization tensor of the GO phase can be determined as follows:

$$
\boldsymbol{A}_{\boldsymbol{i}}=\left[\boldsymbol{I}+f_{m} \boldsymbol{S}: \boldsymbol{C}_{\boldsymbol{m}}^{-1}:\left(\boldsymbol{C}_{\boldsymbol{i}}-\boldsymbol{C}_{\boldsymbol{m}}\right)\right]^{-1}
$$

For isotropic elastic spheres, the Hooke's law can be expressed in terms of stiffness tensor $\boldsymbol{C}_{\boldsymbol{r}=\mathrm{i}, \mathrm{m}}$ and $\boldsymbol{C}_{\boldsymbol{h}}$ as follows: 


$$
\boldsymbol{C}_{\boldsymbol{r}}=3 K_{r} \boldsymbol{R}+2 \mu_{r} \boldsymbol{T}
$$

$$
\boldsymbol{C}_{\boldsymbol{h}}=3 K_{h} \boldsymbol{R}+2 \mu_{h} \boldsymbol{T}
$$

148 where $K_{r=i, m}$ and $\mu_{r=i, m}$ are the bulk moduli and shear moduli of phase inclusion and matrix 149 respectively; $K_{p}$ and $\mu_{p}$ are the homogenized bulk and shear moduli.

150

$151 \boldsymbol{R}$ and $\boldsymbol{T}$ are the volumetric part and the deviator part of the fourth-order unit tensor $\boldsymbol{I}$ respectively 152 and $\boldsymbol{I}=\boldsymbol{R}+\boldsymbol{T}$ where,

$$
\mathbf{R}=R_{i j k l}=1 / 3 \quad \delta_{i j} \delta_{k l}
$$

154

155 as follow,

156

157 where

159 Substituting Equations 8 and 9 and into Equation 7,

160

$$
\boldsymbol{A}_{\boldsymbol{i}}=\frac{1}{f_{m}\left(\alpha \frac{K_{i}}{K_{m}}-\alpha\right)+1} \boldsymbol{R}+\frac{1}{f_{m}\left(\beta \frac{\mu_{i}}{\mu_{m}}-\beta\right)+1} \boldsymbol{T}
$$

161 Combining Equation 9, 10 and 11 leads to the relationship as follows,

$$
\begin{aligned}
& 162 \boldsymbol{C}_{\boldsymbol{h}}=\boldsymbol{C}_{\boldsymbol{m}}+f_{i}\left[\boldsymbol{C}_{\boldsymbol{i}}-\boldsymbol{C}_{\boldsymbol{m}}\right]: \boldsymbol{A}_{\boldsymbol{i}} \\
& 163=\left(3 K_{m} \boldsymbol{R}+2 \mu_{m} \boldsymbol{T}\right)+f_{i}\left[\left(3 K_{i} \boldsymbol{R}+2 \mu_{m} \boldsymbol{T}\right)-\left(3 K_{m} \boldsymbol{R}+2 \mu_{m} \boldsymbol{T}\right)\right]: \boldsymbol{A}_{\boldsymbol{i}} \\
& 164=3 K_{m} \boldsymbol{R}+\frac{f_{i} \cdot 3\left(K_{i}-K_{m}\right)}{\alpha \frac{K_{i}}{K_{m}}-\alpha+1} \boldsymbol{R}+2 \mu_{a} \boldsymbol{T}+\frac{f_{i} \cdot 2\left(\mu_{i}-2 \mu_{m}\right)}{\beta \frac{\mu_{i}}{\mu_{m}}-\beta+1} \boldsymbol{T}
\end{aligned}
$$


And $\boldsymbol{C}_{\boldsymbol{h}}=3 K_{h} \boldsymbol{R}+2 \mu_{h} \boldsymbol{T}$

Hence $3 K_{h} \boldsymbol{R}+2 \mu_{h} \boldsymbol{T}=3 K_{m} \boldsymbol{R}+\frac{f_{i} \cdot 3\left(K_{i}-K_{m}\right)}{\alpha \frac{K_{i}}{K_{m}}-\alpha+1} \boldsymbol{R}+2 \mu_{a} \boldsymbol{T}+\frac{f_{i} \cdot 2\left(\mu_{i}-2 \mu_{m}\right)}{\beta \frac{\mu_{i}}{\mu_{m}}-\beta+1} \boldsymbol{T}$

Therefore, the homogenized bulk elastic modulus $k_{h}$ and shear moduli $\mu_{\mathrm{h}}$ can be determined as follow,

$$
\begin{aligned}
& \mathrm{k}_{\mathrm{h}}=\mathrm{k}_{C S H}+\frac{\mathrm{f}_{G O}\left(\mathrm{k}_{G O}-\mathrm{k}_{C S H}\right)}{1+f_{C S H} \alpha\left(\frac{\mathrm{k}_{G O}}{\mathrm{k}_{C S H}}-1\right)} \\
& \mu_{\mathrm{h}}=\mu_{C S H}+\frac{\mathrm{f}_{G O}\left(\mu_{G O}-\mu_{C S H}\right)}{1+f_{C S H} \beta\left(\frac{\mu_{G O}}{\mu_{C S H}}-1\right)}
\end{aligned}
$$

Finally, the effective Young's modulus after homogenization $\mathrm{E}_{\mathrm{h}}$ can be derived as follows,

$$
\mathrm{E}_{\mathrm{h}}=\frac{9 \mathrm{k}_{\mathrm{h}} \mu_{\mathrm{h}}}{3 \mathrm{k}_{\mathrm{h}}+\mu_{\mathrm{h}}}=\frac{9\left[\mathrm{k}_{C S H}+\frac{\mathrm{f}_{G O}\left(\mathrm{k}_{G O}-\mathrm{k}_{C S H}\right)}{1+f_{C S H} \alpha\left(\frac{\mathrm{k}_{G O}}{\mathrm{k}_{C S H}}\right)}\right] *\left[\mu_{C S H}+\frac{\mathrm{f}_{G O}\left(\mu_{G O}-\mu_{C S H}\right)}{1+f_{C S H} \beta\left(\frac{\mu_{G O}}{\left.\mu_{C S H}-1\right)}\right]}\right.}{3\left[\mathrm{k}_{C S H}+\frac{\mathrm{f}_{G O}\left(\mathrm{k}_{G O}-\mathrm{k}_{C S H}\right)}{1+f_{C S H} \alpha\left(\frac{\mathrm{k}_{G O}}{\mathrm{k}_{C S H}}-1\right)}\right]+\left[\mu_{C S H}+\frac{\mathrm{f}_{G O}\left(\mu_{G O}-\mu_{C S H}\right)}{1+f_{C S H} \beta\left(\frac{\mu_{G O}}{\left.\mu_{C S H}-1\right)}\right.}\right]}
$$

The elastic modulus of C-S-H (i.e., $\mathrm{k}_{\mathrm{h}}$ and $\mu_{\mathrm{h}}$ ) can be obtained from nano-indentation test or molecular dynamics simulation ${ }^{22}$. The modulus of GO (i.e., $\mathrm{k}_{\mathrm{GO}}$ and $\mu_{\mathrm{GO}}$ ) are usually available from commercial producers; it can also be computed or experimentally tested.

The same method can be applied to the next level homogenization for GO-cement paste. As shown in Figure 1b, the cement paste can be considered as a three-phase composite. GO-CSH is treated as the matrix containing calcium hydroxide $(\mathrm{CH})$ and voids $(\mathrm{V})$. Un-hydrated cement is not present in the homogenization process as complete hydration is assumed for water-to-cement (w/c) ratio above 0.4. The homogenized strain can be linked to the individual strain at different phases in terms of the linear strain localization tensors which are shown as follows,

$$
\varepsilon_{r}=\boldsymbol{A}_{r}: \bar{\varepsilon}
$$




$$
\varepsilon_{h}=A_{h}: \bar{\varepsilon}
$$

$$
\varepsilon_{C H}=\boldsymbol{A}_{C H}: \bar{\varepsilon}
$$

$$
\varepsilon_{V}=A_{V}: \bar{\varepsilon}
$$

187 The concentration tensor $\boldsymbol{A}_{\boldsymbol{r}}$ can also be expressed as follows,

$$
<\boldsymbol{A}_{\boldsymbol{r}}>_{V_{p}}=\sum_{r} f_{r}<A_{\boldsymbol{r}}>=\boldsymbol{I}
$$

189 Therefore,

$$
\boldsymbol{A}_{\boldsymbol{h}}=1 / \mathrm{f}_{\boldsymbol{h}}\left[\boldsymbol{I}-\left(\mathrm{f}_{\mathrm{CH}} \boldsymbol{A}_{\boldsymbol{C H}}+\mathrm{f}_{\mathrm{V}} \boldsymbol{A}_{\boldsymbol{V}}\right)\right]
$$

191

192

193

194

195

196

197 follows,

198

199

200

201

$3 K_{u} \boldsymbol{R}+2 \mu_{u} \boldsymbol{T}=\left(3 K_{h} \boldsymbol{R}+2 \mu_{h} \boldsymbol{T}\right)+f_{\boldsymbol{C H}}\left[\frac{3 K_{C H}-3 K_{h}}{f_{m}\left(\alpha \frac{K_{C H}}{K_{h}}-\alpha\right)+1} \boldsymbol{R}+\frac{\left(2 \mu_{C H}-2 \mu_{h}\right)}{f_{m}\left(\beta \frac{\mu_{C H}}{\mu_{h}}-\beta\right)+1} \boldsymbol{T}\right]$

By introducing the linear elastic constitutive relation for different phases contained in $V_{p}$, the linear homogenization formula for the macroscopic elasticity tensor can be determined as follows,

$$
\begin{aligned}
& \boldsymbol{C}_{\boldsymbol{u}}=\left\langle\boldsymbol{C}: \boldsymbol{A}>=\sum_{r} f_{r} \boldsymbol{C}_{r}: \boldsymbol{A}_{\boldsymbol{r}}\right. \\
& =f_{\boldsymbol{h}} \boldsymbol{C}_{\boldsymbol{h}}: \boldsymbol{A}_{\boldsymbol{h}}+f_{\boldsymbol{C H}} \boldsymbol{C}_{\boldsymbol{C H}}: \boldsymbol{A}_{\boldsymbol{C H}}+f_{V} \boldsymbol{C}_{\boldsymbol{V}}: \boldsymbol{A}_{\boldsymbol{V}}
\end{aligned}
$$

The Young's modulus of voids is taken as 0 . For a high w/c ratio of higher than 0.38 , the clinker phase of the unhydrated cement can be ignored. Therefore the above equation can be simplified as $\boldsymbol{C}_{\boldsymbol{h}}+f_{\boldsymbol{C H}}\left[\frac{3 K_{C H}-3 K_{h}}{f_{m}\left(\alpha \frac{K_{C H}}{K_{h}}-\alpha\right)+1} \boldsymbol{R}+\frac{\left(2 \mu_{C H}-2 \mu_{h}\right)}{f_{m}\left(\beta \frac{\mu_{C H}}{\mu_{h}}-\beta\right)+1} \boldsymbol{T}\right]$

$=\left(3 K_{h} \boldsymbol{R}+2 \mu_{h} \boldsymbol{T}\right)+f_{\boldsymbol{C H}}\left[\frac{3 K_{C H}-3 K_{h}}{f_{m}\left(\alpha \frac{K_{C H}}{K_{h}}-\alpha\right)+1} \boldsymbol{R}+\frac{\left(2 \mu_{C H}-2 \mu_{h}\right)}{f_{m}\left(\beta \frac{\mu_{C H}}{\mu_{h}}-\beta\right)+1} \boldsymbol{T}\right]$

Therefore 
follows:

$$
\begin{aligned}
& \mathrm{k}_{\mathrm{u}}=\mathrm{k}_{\mathrm{h}}+\frac{\mathrm{f}_{\mathrm{CH}}\left(\mathrm{k}_{\mathrm{CH}}-\mathrm{k}_{\mathrm{h}}\right)}{1+f_{h} \alpha\left(\frac{\mathrm{k}_{C H}}{\mathrm{k}_{\mathrm{h}}}-1\right)} \\
& \mu_{\mathrm{u}}=\mu_{\mathrm{h}}+\frac{\mathrm{f}_{\mathrm{CH}}\left(\mu_{\mathrm{CH}}-\mu_{\mathrm{h}}\right)}{1+f_{h} \beta\left(\frac{{ }{ }_{C H}}{\mu_{\mathrm{h}}}-1\right)}
\end{aligned}
$$

The homogenized Young's modulus at the macroscale $\mathrm{E}_{\mathrm{u}}$ can be obtained as follows,

$$
\mathrm{E}_{\mathrm{u}}=\frac{9 \mathrm{k}_{u} \mu_{\mathrm{u}}}{3 \mathrm{k}_{\mathrm{u}}+\mu_{\mathrm{u}}}=\frac{9\left(\mathrm{k}_{\mathrm{h}}+\frac{\mathrm{f}_{\mathrm{CH}}\left(\mathrm{k}_{\mathrm{CH}}-\mathrm{k}_{\mathrm{h}}\right)}{1+f_{h} \alpha\left(\frac{\mathrm{k}_{C H}}{\mathrm{k}_{\mathrm{h}}}-1\right)}\right)\left(\mu_{\mathrm{h}}+\frac{\mathrm{f}_{\mathrm{CH}}\left(\mu_{\mathrm{C}}-\mu_{\mathrm{h}}\right)}{1+f_{h} \beta\left(\frac{{ }{ }_{C H} \mu_{\mathrm{h}}}{\mu_{\mathrm{h}}}-1\right)}\right)}{3\left(\mathrm{k}_{\mathrm{h}}+\frac{\mathrm{f}_{\mathrm{CH}}\left(\mathrm{k}_{\mathrm{CH}}-\mathrm{k}_{\mathrm{h}}\right)}{1+f_{h} \alpha\left(\frac{\mathrm{k}_{C H}}{\mathrm{k}_{\mathrm{h}}}-1\right)}\right)+\left(\mu_{\mathrm{h}}+\frac{\mathrm{f}_{\mathrm{CH}}\left(\mu_{\mathrm{CH}}-\mu_{\mathrm{h}}\right)}{1+f_{h} \beta\left(\frac{\mu_{C H} C H}{\mu_{\mathrm{h}}}-1\right)}\right)}
$$

Equation (27) shows the Young's modulus of GO-cement at macroscale as a function of a number of parameters at different length scales. All the key material and geometric factors have been considered in this formulation. The elastic modulus of $\mathrm{CH}$ can be obtained from experimental test or molecular dynamics simulation. The volume fraction of voids can be estimated from porosity usually obtained from MIP test.

\section{EXPERIMENTAL PROGRAM}

As shown in Figure 4(a), in-situ SEM three-point bending tests were performed on beams of $6 \mathrm{~mm}$ $\times 10 \mathrm{~mm} \times 40 \mathrm{~mm}$ using Tungsten Filament Scanning Electron Microscope (SEM) incorporated with a three-point bending testing mechanism to determine their Young's modulus and fracture mechanisms. A notch of $2 \mathrm{~mm}$ was placed in the middle of each beam. A commercially available GO solution with a GO concentration $4 \mathrm{mg} / \mathrm{ml}$ was used in this investigation. The chemical composition of GO provided by the manufacturer is Carbon $49-56 \%$, Hydrogen $0-1 \%$, Nitrogen $0-1 \%$, Sulfur 0-2\% and Oxygen 41-50\%. GO-cement composites with w/c $=0.4$ were prepared by mixing cement, water and GO. The dosages of GO were $0.00 \%, 0.02 \%$, and $0.035 \%$ by weight. 
These dosages were obtained by adjusting the concentration of the as received GO solution. For each GO dosage, the required amount of water was added to the GO solution and the resulting solution was sonicated for 30 minutes using a bath sonicator. The GO-cement composites were prepared by simply mixing the GO solution and the cement powder in a mixer for 3 minutes. The fresh GO-cement composite was then poured into a $6 \mathrm{~mm} \times 10 \mathrm{~mm} \times 40 \mathrm{~mm}$ moulds and kept for 24 hours at room temperature. The samples were then de-moulded and cured under water for 28 days at temperature of $20^{\circ} \mathrm{C} .3$ specimens were produced for each batch. The samples were subjected to load in a displacement-control mode with a speed of $0.05 \mathrm{~mm} / \mathrm{min}$. The load-deflection curves were recorded and used to determine the Young's modulus and morphology of the GO-cement composites at different scales. The testing arrangement and the geometric parameters are illustrated in Figure 4. The morphology of the GO-cement composites was examined with the SEM. In addition, porosity tests (MIP) were conducted on broken samples obtained from the micro three-point bending test to determine the effect of GO on the porosity of the GO-cement composites.

\section{EXPERIMENTAL RESULTS AND DISCUSSION}

Figure 5 shows the load-deflection curves obtained from the micro three-point bending tests. As in this figure, the flexural response of the composites can be divided into three parts: elastic deformation, plastic deformation and fracture stage. Figure 5 indicates that the failure load of the composites increases with increasing GO dosage. This is due to the microscale enhancement of cement by the GO through crack bridging mechanism, cement porosity reduction, chemical reaction between the functional groups of GO and cement to form stronger interfaces, etc.

The microstructures of the GO-cement with different GO dosages are presented in Figure 6. All samples have shown considerable amount of needle-like Ettringite minerals. The GO wrapped with 
cement has been identified on the surface of the sample with $0.035 \%$ and $0.06 \%$ GO inclusions by the SEM test. It has been found that the fracture surface for pure cement is relatively smoother, whilst the $0.035 \%$ GO-cement sample shows rougher surface, especially around the GO, whose length ranges from $3 \mu \mathrm{m}$ to $30 \mu \mathrm{m}$ in its presence of GO-cement. It is believed that the existence of GO in cement can change the hydration process or packing of cement particles. Due to the functional groups attached, GO tends to react with the $\mathrm{C}-\mathrm{S}-\mathrm{H}$, the main binding phase of the cement, forming chemically-bound interface between oxygen in GO and calcium in cement ${ }^{23}$. Such a chemically-bound cementitious nanocomposite can provide higher stress transfer through the interfaces and hence stronger strength of the material. Moreover, Figure 7 illustrates the fracture process obtained for the $0.035 \% \mathrm{GO}$-cement sample from the micro three-point bending test. The crack is developed as the loading increases. The fracture process shows some kind of shielding effect, since the fracture path is tortuous. The loads are in Figure 7 for each stage of the crack and all these loads are in the softening curve of the load-deflection relationship in Figure 5. The results obtained from the porosity tests indicate that GO decreases the porosity of the cementitious composites. For example, the percentage of porosity decreased from $15.57 \%$ at a GO dosage of $0-\mathrm{wt} \%$ to $14.56 \%$ at a GO dosage of $0.035-\mathrm{wt} \%$. This is due the fact that GO tends to amplify the hydration products, and fill and cover the pores in the matrix, thus densifying the cement pastes with less porosity. This is consistent with results reported in ${ }^{10,11}$.

\section{Model Verification}

To verify the developed analytical model, the results are compared with those from the experiments. The measured Young's modulus of the GO-cement composite was determined using the elastic deformation phase, can be calculated as follows ${ }^{24}$ :

$$
\mathrm{E}=\frac{P_{a} D^{3}}{4 t\left(b-a_{0}\right)^{3} d}
$$


where $P_{a}$ is the load within the elastic stage, $D$ is the distance between the two supports, $t$ is the width of the specimen, $b$ is the depth of specimen, $\mathrm{a}_{0}$ is the notch and $d$ is the deflection of specimen.

The Young's modulus of the GO-cement composites was predicted using the materials properties given in Table 1. The predicted and measured Young's modulus of the GO-cement composites are given in Table 2. Table 2 shows that the predicted results are in a good agreement with the experimental results. For $0.02-\mathrm{wt} \%$ of $\mathrm{GO}$ inclusion by weight, the Young's modulus of cement determined from the test increases from $11.63 \mathrm{GPa}$ to $12.63 \mathrm{GPa}$ and from the model increases to 12.99GPa. The enhancement in Young' modulus is $8.6 \%$ and $11.7 \%$ respectively. The difference could be partially due to the assumption that the interfaces between GO and cement are perfected bonded. This means there is no slip at the interfaces and thus loss in stress transfer between these two materials is minimal. This leads to slightly higher Young's modulus in numerical results. However, for $0.035-\mathrm{wt} \%$ of GO inclusion, the Young's modulus from both the experiment and the model agree very well. Moreover, it is very interesting to see that the relationship between the increase of GO dosage and the enhancement of Young's modulus is not proportional; the increase of Young's modulus of GO-cement is much faster than that of GO dosage.

\section{Parametric Study}

One of the advantages of the analytical model is that the effects of a number of factors can be investigated. In this paper a few key parameters were chosen from the derived analytical model to examine the sensitivity of those parameters to the elasticity of GO-cement. From the model, the volume fractions and Young's modulus for GO, C-S-H and $\mathrm{CH}$ were selected, as well as the volume fraction of void, in order to study their effects on the homogenized Young's modulus of GO-cement. Figure 8 presents the macro scale Young's modulus of GO-cement as a function of Young's 
modulus of GO. It can be seen that the Young's modulus of GO-cement increases as the Young's modulus of GO increases. Three different percentages of GO addition are also plotted in the figure. As expected, the increase in volume fraction of GO can also increase the homogenized Young's modulus of the GO-cement. The increase is roughly in linear relationship. It is interesting to see that the Young's modulus of GO does not change the macroscope Young's modulus too much for the same volume fraction of the GO inclusion investigated; but higher volume fraction of GO can increase the elastic modulus of the GO-cement a lot more. This demonstrates that for a very small amount of GO inclusion in cement, the mechanical property of the inclusion is less important. Nevertheless, compared with the normal cement, the Young's modulus of GO-cement can still be enhanced significantly for a small GO inclusion.

Figure 9 illustrates the effects of the volume fraction as well as the elastic modulus of C-S-H on the global elastic modulus of the GO-cement. It can be seen that the Young's modulus of GO-cement decreases with the increase of the volume fraction of C-S-H. The reason is because the matrix phase of C-S-H has lower Young's modulus than its inclusion phase GO; the increase in the volume of $\mathrm{C}-\mathrm{S}-\mathrm{H}$ would decrease the volume of the GO and hence the decrease in global elastic modulus. Meanwhile, the increase in the Young's modulus of the C-S-H can significantly increase of the Young's modulus of the GO-cement, as shown in Figure 9. The Young's modulus of C-S-H is investigated between 15 and $45 \mathrm{GPa}$ since it is the range that the literature suggests. It can be seen that the overall Young's modulus is very sensitive to that of C-S-H. This can be easily understood since $\mathrm{C}-\mathrm{S}-\mathrm{H}$ is the dominate phase material in the composite. Figure 10 shows the relationship between the homogenized elastic modulus of the GO-cement and that of the $\mathrm{CH}$ phase, as well as its volume. The homogenized Young's modulus of GO-cement can increase as either the Young's modulus of $\mathrm{CH}$ rises or the volume fraction of $\mathrm{CH}$ does. 
As discussed, GO in the cement composites can be in the form of either folding or stacking. These two mechanisms are not separately considered in the analytical model for the homogenized elastic property of GO-cement. This is because the homogenization scheme employed in this study only allows one inclusion phase. As such, a single phase of GO is assumed in the modeling. However, the mechanical properties of the folding GO and the stacking GO are not identical and to roughly estimate the effects of different volumes of these two types of GO, Figure 11 is plotted. The simple weighted average method was employed for the estimation. The assumption is based on that the Young's modulus of the folding and stacking GO are $350 \mathrm{GPa}$ and $300 \mathrm{GPa}$, respectively. These values are estimated since there are no experimental results yet. It can be seen that with the increase of the volume fraction of the relevant $\mathrm{GO}$, the elastic modulus for both scenarios increase and the increase for the folding GO is higher than that of stacking GO, as expected. The difference between the two scenarios is significant for larger fraction of GO inclusion while relatively small for low GO fraction.

\section{CONCLUSIONS}

In this paper a multiscale analytical model has been derived for the elastic modulus of the GO-cement. Young's modulus and volume fractions for different composition at different scales, i.e., micro, meso and macro scales, are formulated in the developed model. In-situ three-point bending SEM test was also undertaken to determine the Young's modulus of GO-cement experimentally and the results have been compared with the analytical model. It has been found that the results from the derived model and the experimental tests are in good agreement. Moreover, the in-situ SEM tests have shown the crack propagation process and the cracking bridging mechanism was observed and discussed. MIP test has also been conducted to determine the porosity of the GO-cement with different concentrations. It can be concluded that the model developed is perhaps the only analytical model that can predict the elastic mechanical performance of GO-cement by considering all 
347 possible scales. The addition of GO can significantly change the morphology and substantially 348 enhance the mechanical properties of the cement with respect to the amount of GO added. The 349 developed model can be used as a useful tool to evaluate the global elastic mechanism of 350 GO-cement based on the microscale mechanical behavior of the materials.

351

\section{ACKNOWLEDGMENTS}

353 Partial finance support from European Commission Horizon 2020 Marie Sklodowska-Curie 354 Research and Innovation Staff Exchange scheme through the grant 645696 (i.e. REMINE project) 355 and from EPSRC under EP/L014041/1 is gratefully acknowledged. 
1. Chen J, Kou S-c and Poon C-s. Hydration and properties of nano-TiO 2 blended cement composites. Cement and Concrete Composites. 2012; 34: 642-649.

359 2. Onuaguluchi O, Panesar DK and Sain M. Properties of nanofibre reinforced cement composites. Construction and 360 Building Materials. 2014; 63: 119-124. 3. Siddique R and Mehta A.
Materials. 2014; 50: 116-129.

4. Saafi M, Tang L, Fung J, Rahman M and Liggat J. Enhanced properties of graphene/fly ash geopolymeric composite cement. Cement and Concrete Research. 2015; 67: 292-299.

5. Hancock Y. The 2010 Nobel Prize in physics-ground-breaking experiments on graphene. Journal of Physics D: Applied Physics. 2011; 44: 473001.

6. Yang X, Tu Y, Li L, Shang S and Tao X-m. Well-dispersed chitosan/graphene oxide nanocomposites. ACS applied materials \& interfaces. 2010; 2: 1707-1713.

7. Hashin Z. Analysis of composite materials-a survey. Journal of Applied Mechanics. 1983; 50: 481-505.

8. Qiu L, Yang X, Gou X, et al. Dispersing carbon nanotubes with graphene oxide in water and synergistic effects between graphene derivatives. Chemistry-A European Journal. 2010; 16: 10653-10658.

9. Lv S, Ting S, Liu J and Zhou Q. Use of graphene oxide nanosheets to regulate the microstructure of hardened cement paste to increase its strength and toughness. CrystEngComm. 2014; 16: 8508-8516.

10. Li W, Li X, Chen SJ, Liu YM, Duan WH and Shah SP. Effects of graphene oxide on early-age hydration and electrical resistivity of Portland cement paste. Construction and Building Materials. 2017; 136: 506-514.

11. Li X, Liu YM, Li WG, et al. Effects of graphene oxide agglomerates on workability, hydration, microstructure and compressive strength of cement paste. Construction and Building Materials. 2017; 145: 402-410.

12. Rafiee $\mathrm{R}$ and Firouzbakht V. Multi-scale modeling of carbon nanotube reinforced polymers using irregular tessellation technique. Mechanics of Materials. 2014; 78: 74-84.

13. Shokrieh MM and Rafiee R. Stochastic multi-scale modeling of CNT/polymer composites. Computational Materials Science. 2010; 50: 437-446.

14. Rafiee R. Influence of carbon nanotube waviness on the stiffness reduction of CNT/polymer composites. Composite Structures. 2013; 97: 304-309.

15. Zhang $\mathrm{J}$ and Jiang $\mathrm{D}$. Molecular dynamics simulation of mechanical performance of graphene/graphene oxide paper based polymer composites. Carbon. 2014; 67: 784-791.

16. Hashin Z. The elastic moduli of heterogeneous materials. Journal of Applied Mechanics. 1962; 29: 143-150.

17. Kouznetsova V, Brekelmans W and Baaijens F. An approach to micro-macro modeling of heterogeneous materials. Computational Mechanics. 2001; 27: 37-48.

18. Alkhateb H, Al-Ostaz A, Cheng AH-D and Li X. Materials genome for graphene-cement nanocomposites. Journal of Nanomechanics and Micromechanics. 2013; 3: 67-77.

19. Noh YJ, Joh H-I, Yu J, et al. Ultra-high dispersion of graphene in polymer composite via solvent free fabrication and functionalization. Scientific Reports. 2015; 5: 9141.

20. Eshelby JD. The determination of the elastic field of an ellipsoidal inclusion, and related problems. Proceedings of the Royal Society of London A: Mathematical, Physical and Engineering Sciences. The Royal Society, 1957, p. 376-396.

21. Mori T and Tanaka K. Average stress in matrix and average elastic energy of materials with misfitting inclusions. Acta metallurgica. 1973; 21: 571-574.

22. Pellenq RJ-M, Kushima A, Shahsavari R, et al. A realistic molecular model of cement hydrates. Proceedings of the National Academy of Sciences. 2009; 106: 16102-16107.

23. Pan Z, He L, Qiu L, et al. Mechanical properties and microstructure of a graphene oxide-cement composite. Cement and Concrete Composites. 2015; 58: 140-147.

24. Hibbeler RC. Mechanics of Materials. Macmillan College Pub., 1994. 
404 LIST OF TABLES

405 1. Material properties for GO and C-S-H as input for the model

406 2. Comparison of the results from the experiments and the model 
407 Table 1: Material properties for GO and C-S-H as input for the model

\begin{tabular}{cccc}
\hline Properties & GO & C-S-H & CH \\
\hline Young's modulus E & $350 \mathrm{GPa}$ & $24 \mathrm{GPa}$ & $38 \mathrm{GPa}$ \\
Poisson's ratio v & 0.197 & 0.24 & 0.31 \\
\hline
\end{tabular}

408

409 
410 Table 2: Comparison of the results from the experiments and the model

\begin{tabular}{ccccc}
\hline \multicolumn{2}{c}{ Weight fraction of GO } & $\mathbf{0 \%}$ & $\mathbf{0 . 0 2 \%}$ & $\mathbf{0 . 0 3 5 \%}$ \\
\hline \multirow{2}{*}{ Experiment } & Young's Modulus & $11.63 \mathrm{GPa}$ & $12.63 \mathrm{GPa}$ & $14.64 \mathrm{GPa}$ \\
& Improvement & n.a. & $8.6 \%$ & $25.9 \%$ \\
\multirow{2}{*}{ Model } & Young's Modulus & $\begin{array}{c}11.63 \mathrm{GPa} \\
\text { (assuming) }\end{array}$ & $12.99 \mathrm{GPa}$ & $14.89 \mathrm{GPa}$ \\
& Improvement & n.a. & $11.7 \%$ & $28.0 \%$ \\
\hline
\end{tabular}


413 1. The scale range of GO-cement (a) microscale (b) mesoscale (c) macroscale

414 2. The schematic of folding GO and stacking GO in C-S-H

415 3. The proposed RVE and the upscaling process

416 4. The schematic of three point bending test

417 5. Load-deflection curves achieved from three-point bending tests

418 6. SEM pictures of (a) $0.035 \%$ GO-cement and (d) $0.06 \%$ GO-cement

419 7. Fracture process of the sample of $0.035 \%$ GO-cement

420 8. Relationship between Young's modulus of GO and the Young's modulus of GO-cement

421 9. Relationship between Young's modulus of C-S-H and the Young's modulus of GO-cement

422 10. Relationship between Young's modulus of $\mathrm{CH}$ and the Young's modulus of GO-cement

423 11. Relationship between the volume fraction of two different existences of GO and Young's 424 modulus of GO-cement 


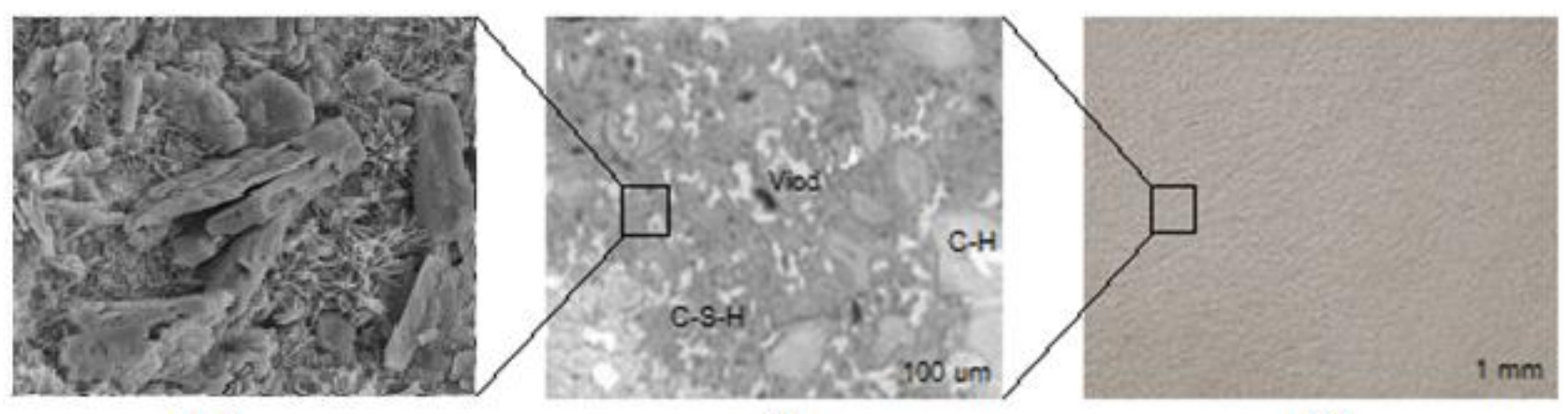

Figure 1 The scale range of GO-cement (a) microscale (b) mesoscale (c) macroscale 


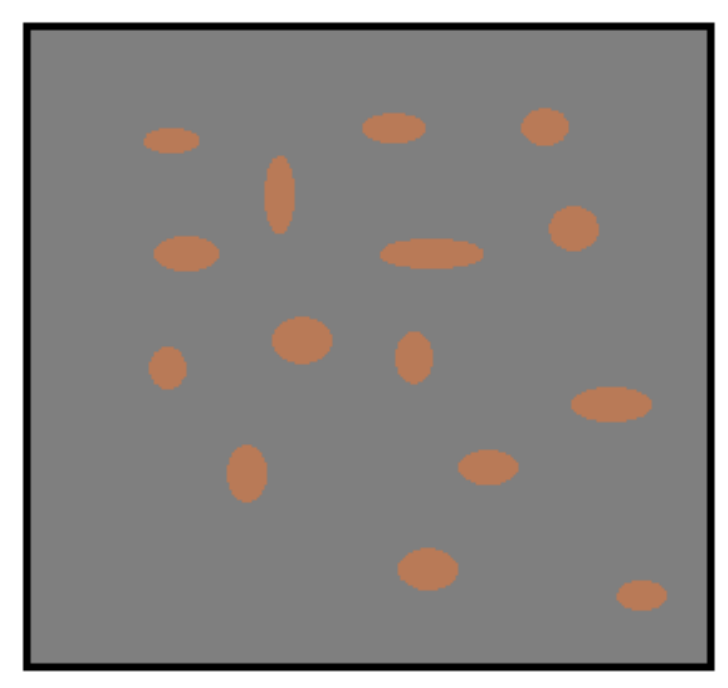

Folding GO

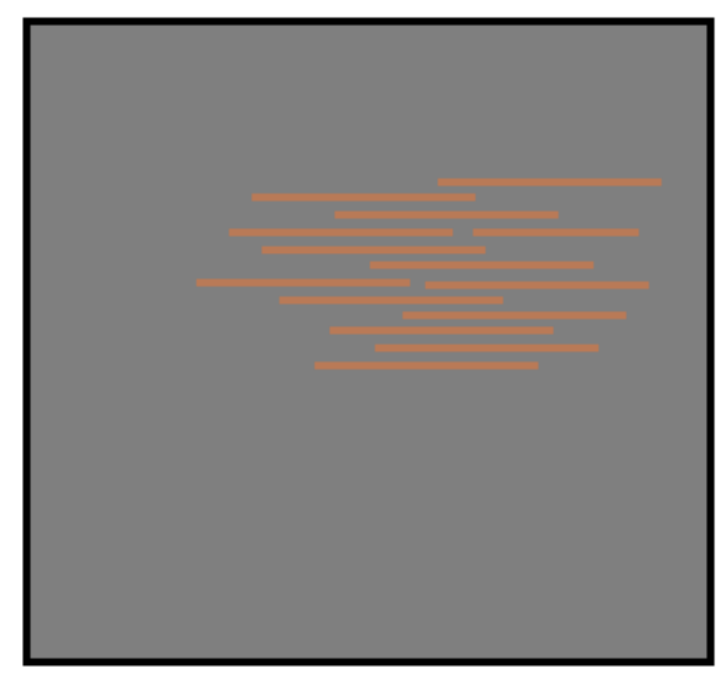

Stacking GO

432

Figure 2 The schematic of folding GO and stacking GO in C-S-H

434 

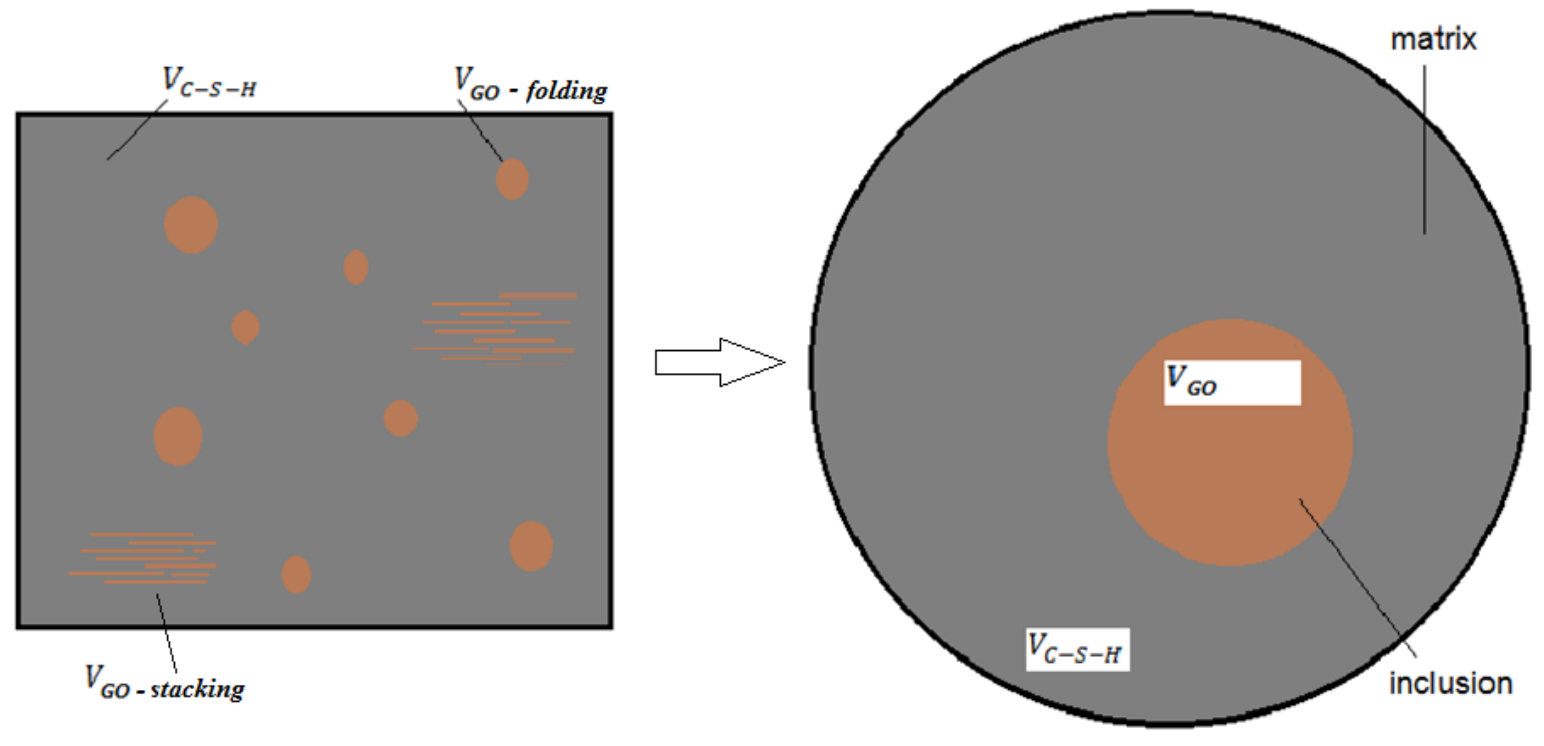

436

Figure 3 The proposed RVE and the upscaling process 

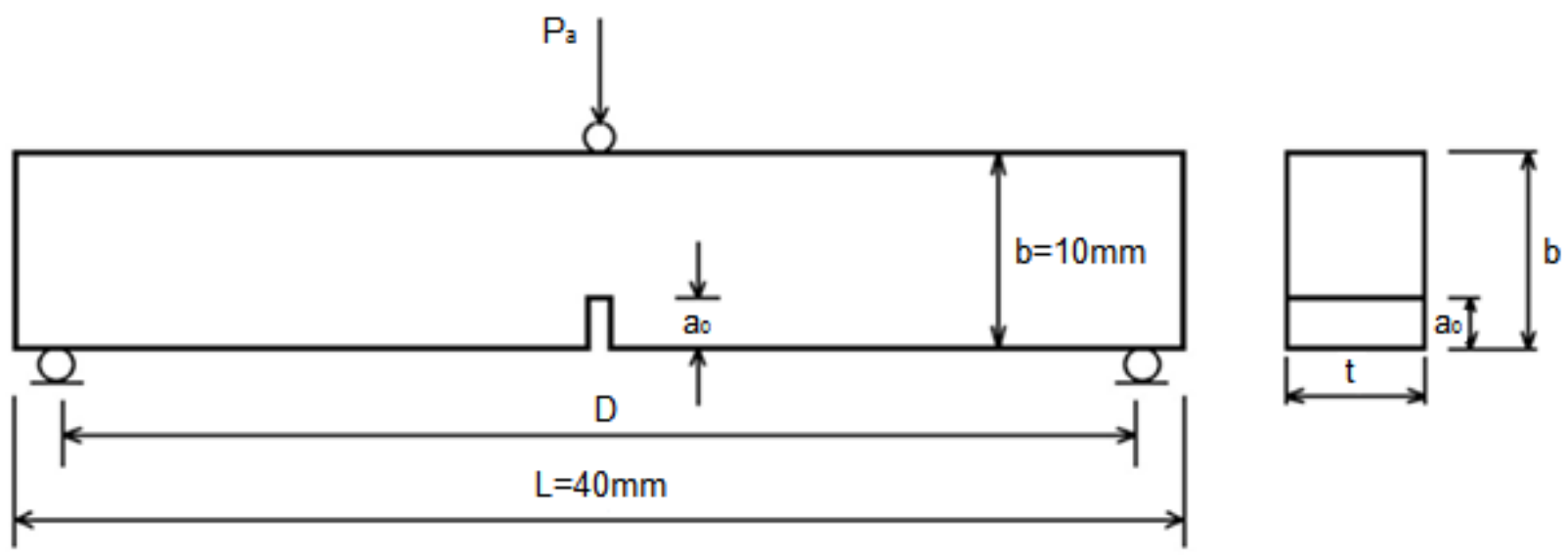

Figure 4 The schematic of three-point bending test 


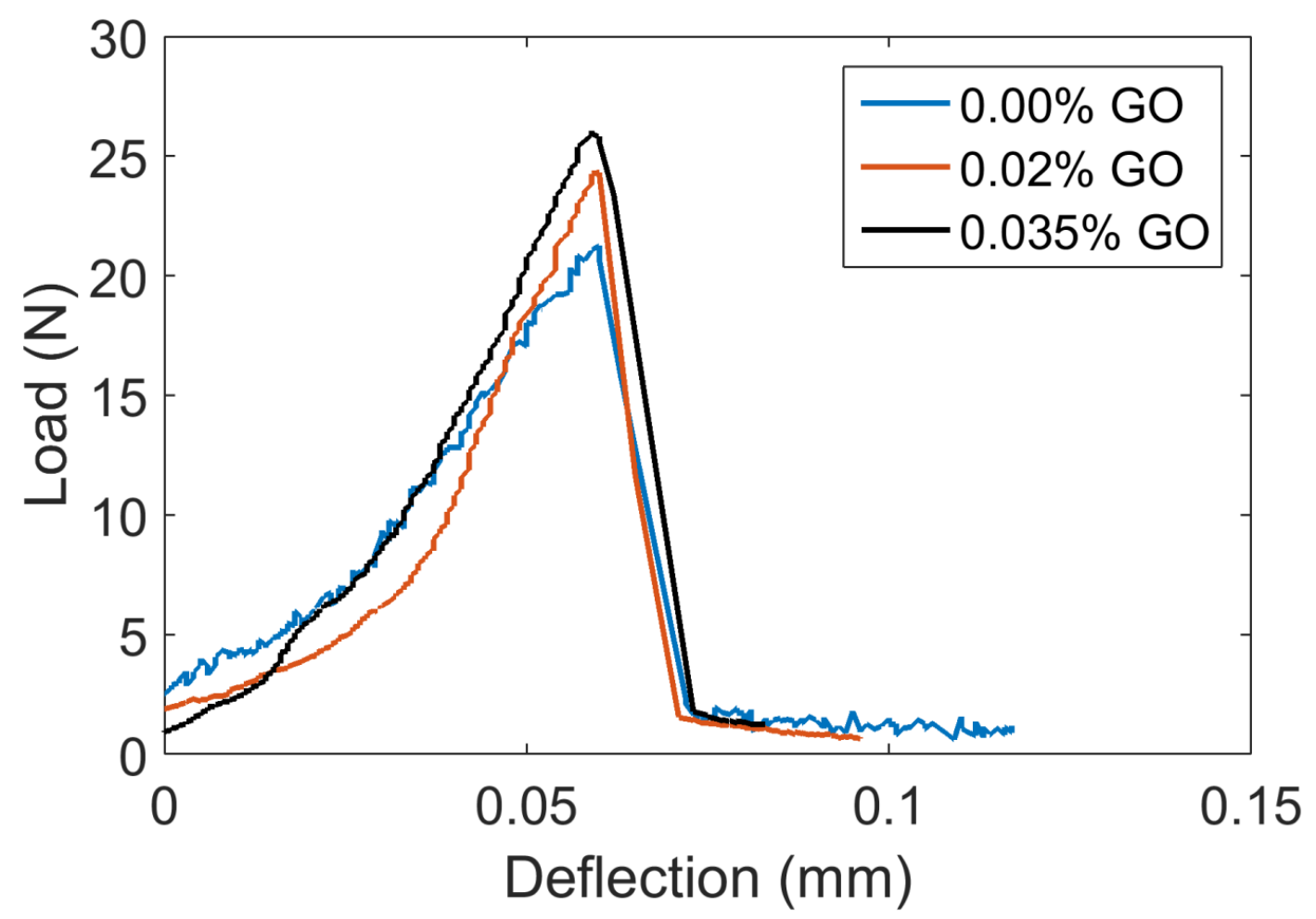

Figure 5 Load-deflection curves obtained from the three-point bending tests 


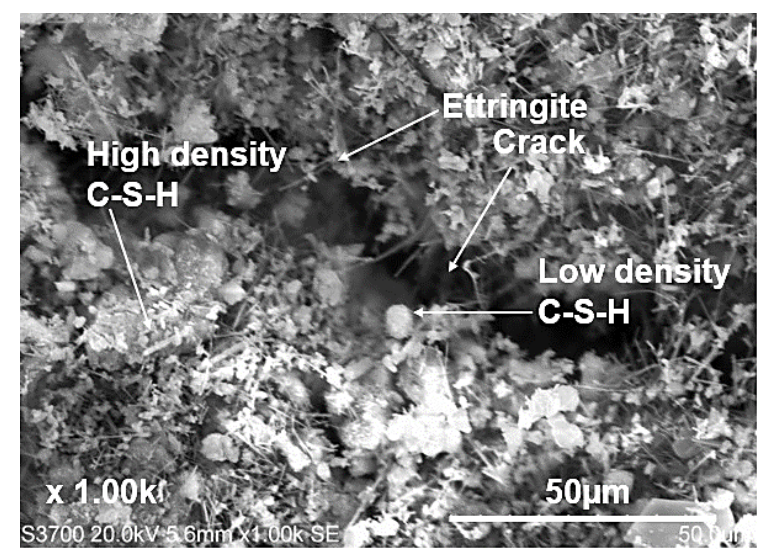

(a)

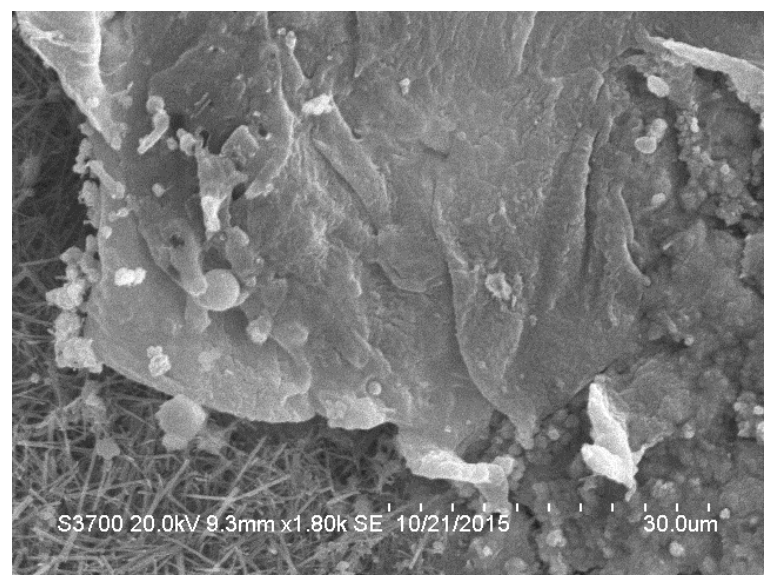

(c)

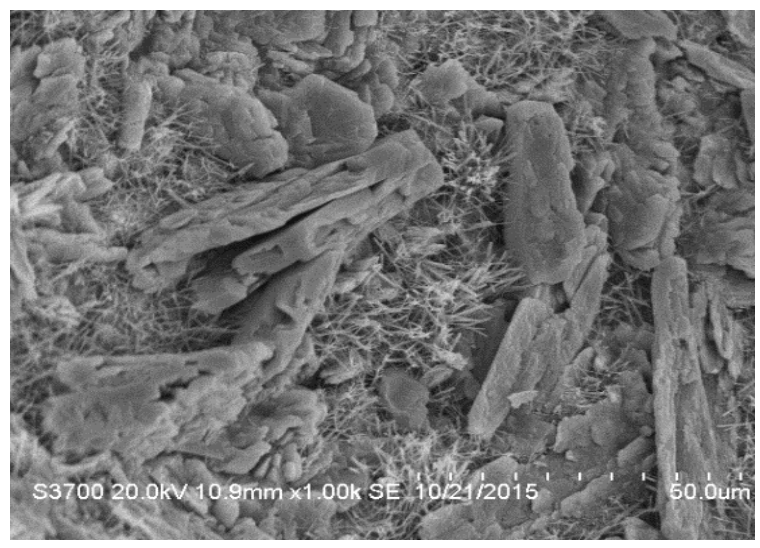

(b)

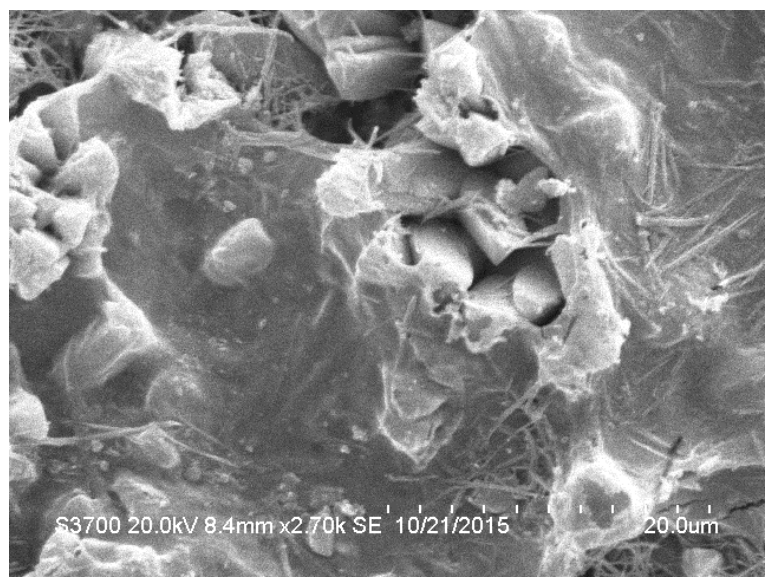

(d)

443 Figure 6 SEM pictures of SEM pictures of (a) $0 \%$ GO-cement (b) $0.02 \%$ GO-cement, (c) $0.035 \%$ 


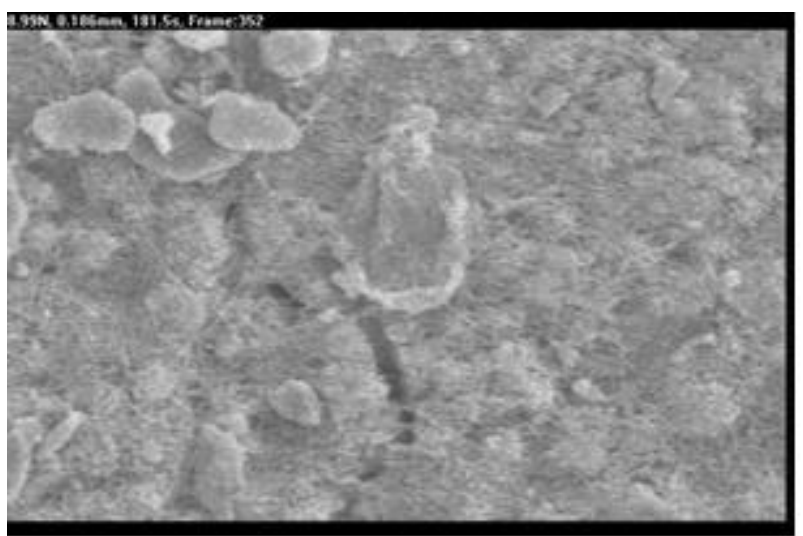

(a) $5.95 \mathrm{~N}$

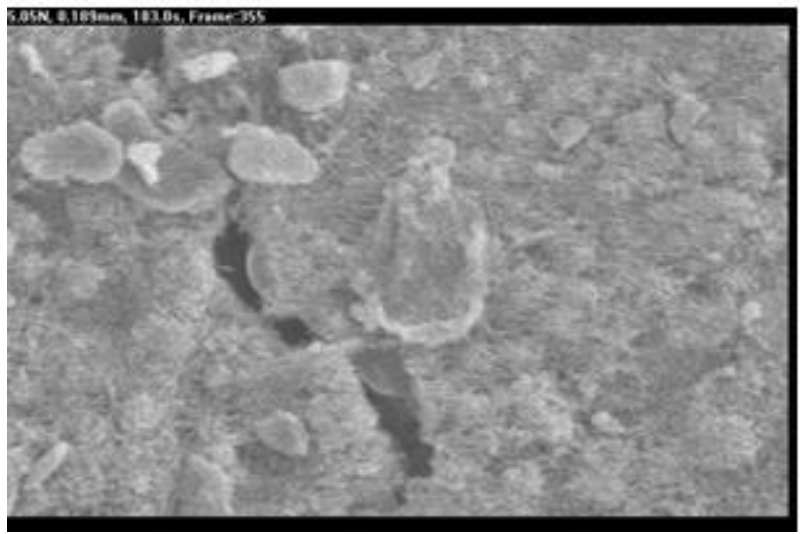

(c) $5.05 \mathrm{~N}$

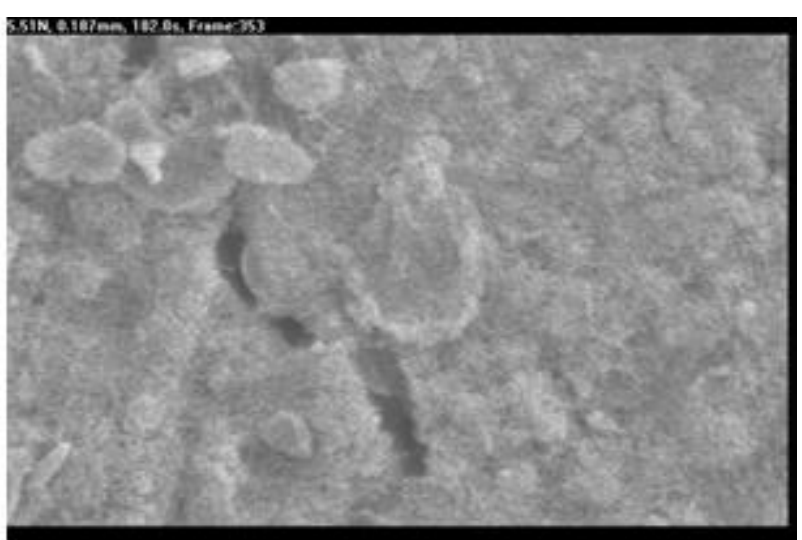

(b) $5.51 \mathrm{~N}$

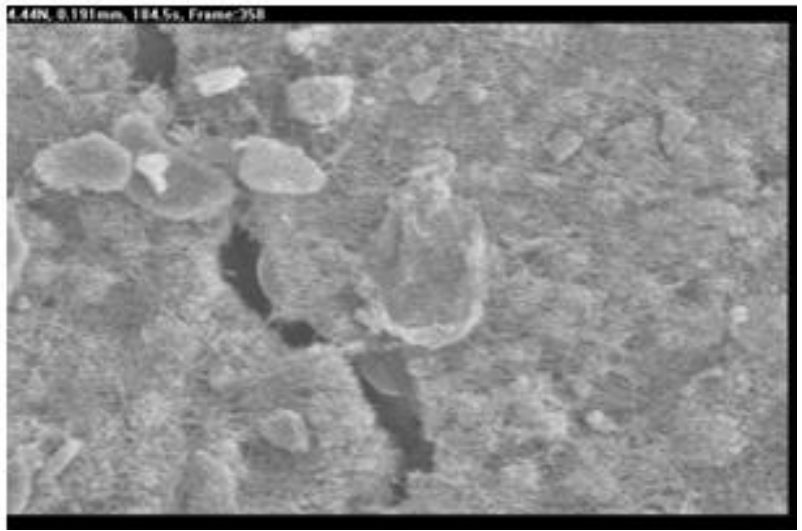

(d) $4.44 \mathrm{~N}$ 
451

452

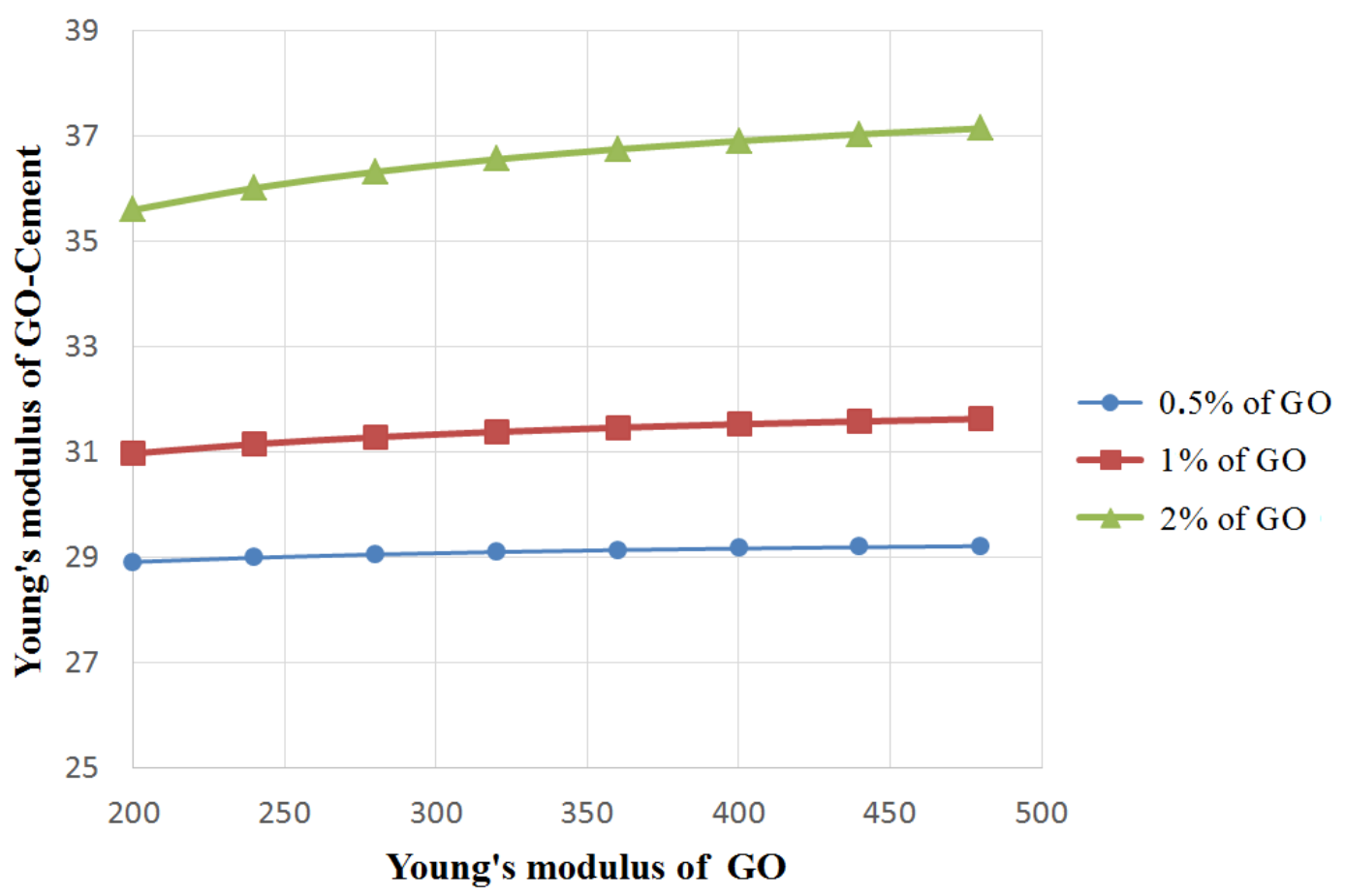

453

454 Figure 8 Relationship between Young's modulus of GO and Young's modulus of GO-cement 


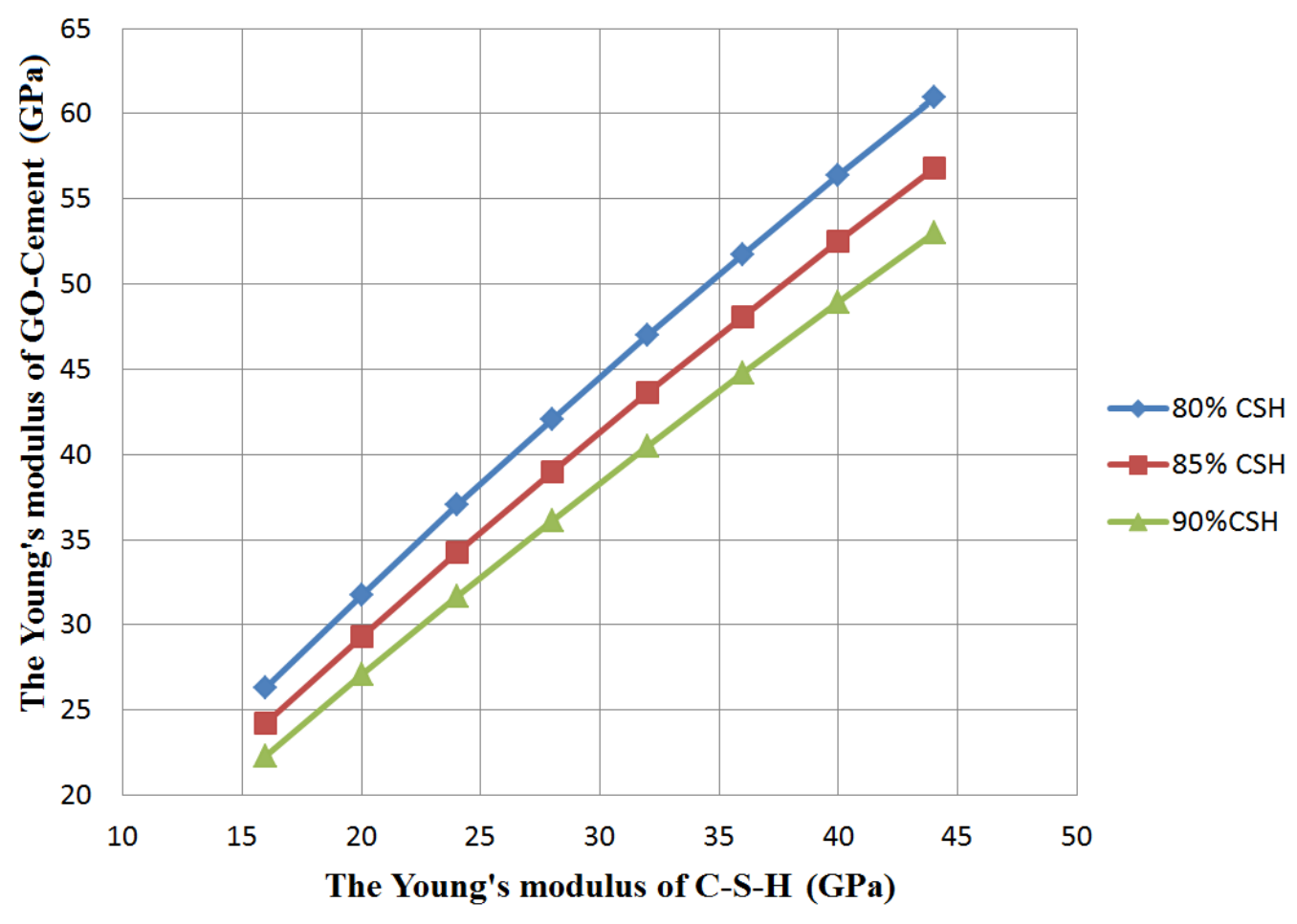

457 Figure 9 Relationship between Young's modulus of C-S-H and Young's modulus of GO-cement 458 


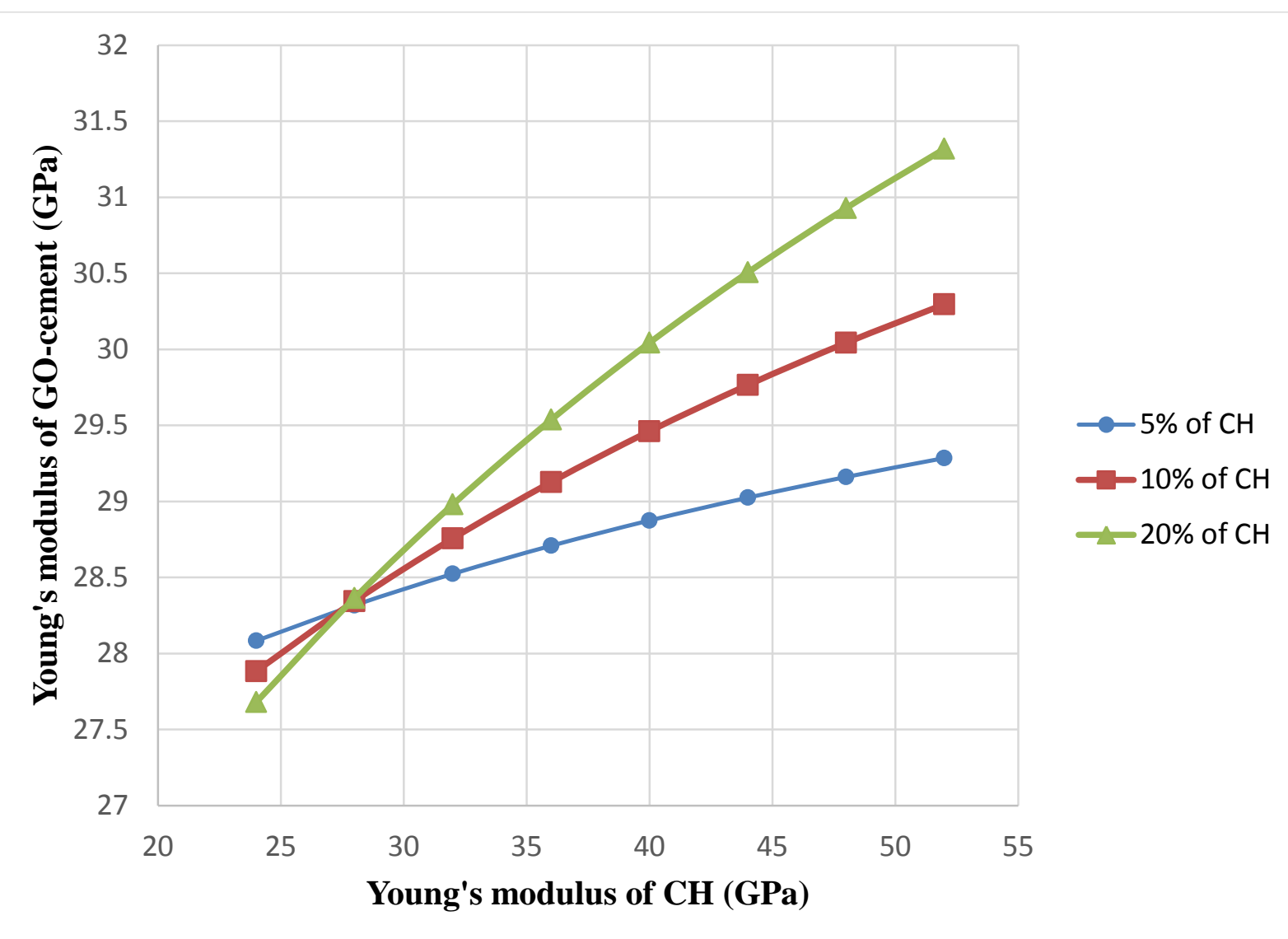

460

Figure 10 Relationship between Young's modulus of $\mathrm{CH}$ and Young's modulus of GO-cement 


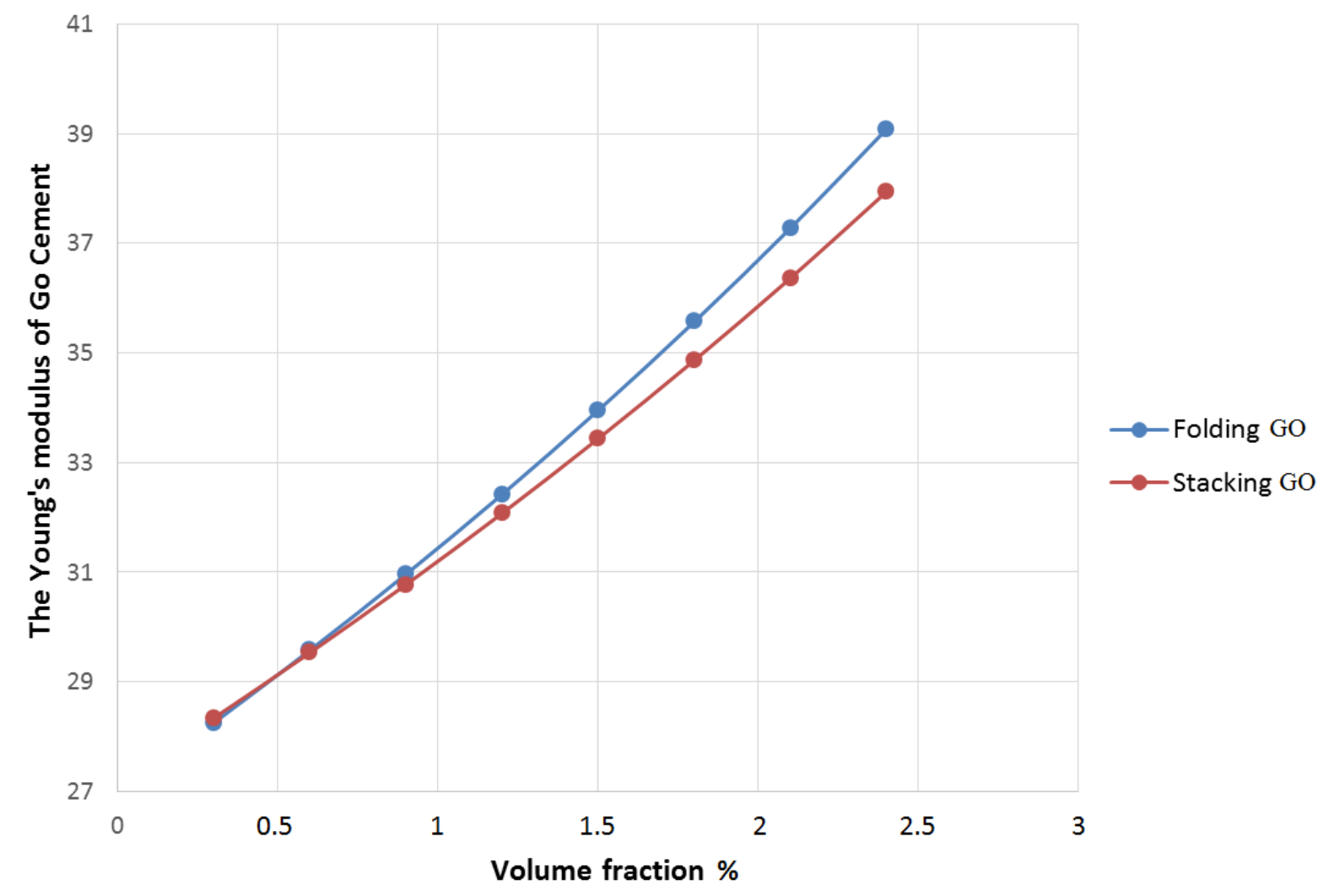

464 Figure 11 Relationship between the volume fraction of two different existences of GO and Young's 